\title{
ENDOMORPHISM RINGS GENERATED BY UNITS $\left(^{1}\right)$
}

\author{
BY \\ PAUL HILL
}

1. The structure of the endomorphism ring of a primary abelian group was studied by Pierce in [6] and [7]. Later, Stringall [8] considered the specific problem of describing those primary groups which have the property that their endomorphism rings are generated additively by automorphisms. For a primary group $G$ having no elements of infinite height, Stringall actually gave necessary and sufficient conditions for the endomorphism ring of $G$ to be generated by automorphisms. One of Stringall's conditions is that each isomorphism of $G$ into $G$ be expressible as the sum of automorphisms of $G$. There are, of course, groups trivially satisfying this condition because, for example, each isomorphism of $G$ into $G$ is already itself an automorphism of $G$; however, in general the question of whether or not each isomorphism of $G$ into $G$ is the sum of automorphisms seems to be roughly the same order of difficulty as the original problem of whether each endomorphism of $G$ is the sum of automorphisms. Thus Stringall's work does not answer in a completely satisfactory manner, even for groups without elements of infinite height, the following problem. For which primary groups is the endomorphism ring generated by automorphisms? One of the main results of this paper is that all totally projective $p$-groups, with $p \neq 2$, have this property. This extends a recent theorem of Castagna [1], who proved the result for totally projective groups having length not exceeding a certain bound, in particular, for direct sums of countable groups. Freeman established the result in [2] for direct sums of cyclic groups.

Throughout, $p$ denotes a fixed prime and primary means $p$-primary. Define $G[p]=[x \in G: p x=0]$ and $p G=[p x: x \in G]$. For each ordinal $\alpha$, define $p^{\alpha} G$ inductively by $p^{\alpha+1} G=p\left(p^{\alpha} G\right)$ and $p^{\beta} G=\bigcap_{\alpha<\beta} p^{\alpha} G$ if $\beta$ is a limit ordinal. Notationally, the operator $p^{\alpha}$ takes priority over $[p]$, that is, $p^{\alpha} G[p]$ means $\left(p^{\alpha} G\right)[p]$. If $G$ is a reduced primary group, there exists an ordinal $\alpha$ such that $p^{\alpha} G=0$. The smallest such ordinal $\alpha$ is called the length of $G$. The height in $G$ of an element $x$ of $G$ is denoted by $h_{G}(x)$ and is defined to be the largest ordinal $\alpha$ such that $x \in p^{\alpha} G$; if no such $\alpha$ exists, then we let $h_{G}(x)=\infty$. If $G$ is a reduced primary group, then $h_{G}(x)=\infty$ only if $x=0$.

2. The endomorphism ring of $G$ is denoted by $E(G)$ and $A(G)$ denotes the automorphism group of $G$. The subring of $E(G)$ generated additively by $A(G)$ is denoted by $\{A(G)\}$. We shall be interested in extending automorphisms of subgroups of $G$ to automorphisms of $G$. In order to have any chance of extending an automorphism $\pi$

Presented to the Society, November 9, 1968; received by the editors September 16, 1968.

( ${ }^{1}$ This work was supported by NSF Grant GP 7442 and NSF Grant GP 8833. 
of a subgroup $H$ of $G$ to an automorphism of $G$, obviously the following condition must be satisfied: $h_{G}(\pi(x))=h_{G}(x)$ for all $x$ in $H$. If this condition is satisfied, we say that $\pi$ preserves heights (in $G$ ) -the parenthetical phrase is usually deleted when the meaning is clear.

The fact that $E(G)=\{A(G)\}$ for a finite commutative group $G$ of odd order is a corollary of the following theorem.

THEOREM 2.1. Let $G$ be a p-primary abelian group with $p>2$ and let $\alpha$ be an ordinal number. Suppose that $A$ is a subgroup of $G$ such that $\left\{p^{\alpha} G, A\right\} \mid A$ is finite. Let $\varphi$ be an endomorphism of $G$ that maps $A$ into $A$. If $\pi$ and $\chi$ are automorphisms of $A$ that preserve heights in $G$ and if their sum is $\varphi$ on $A$, then $\pi$ and $\chi$ can be extended to height-preserving isomorphisms of $\left\{p^{\alpha} G, A\right\}$ in such a way that their sum is still $\varphi$ on $\left\{p^{\alpha} G, A\right\}$.

Proof. There is nothing to prove if $p^{\alpha} G \subseteq A$, so we assume that $p^{\alpha} G$ is not contained in $A$. Let $n$ be the smallest nonnegative integer such that $p^{\alpha+n+1} G \subseteq A$. Let $p^{\alpha+n} G[p]=\left(p^{\alpha+n} G[p] \cap A\right)+S$. Observe that $\left\{p^{\alpha+n} G[p], A\right\}=A+S$. Since $\varphi$ maps $A$ into $A$ and since $p^{\alpha+n} G[p]$ is a fully invariant subgroup of $G, \varphi$ must map $A+S$ into itself. Define $\bar{\varphi}$ on $S$ to be the composite map $\theta \varphi$ where $\theta$ is the natural projection of $A+S$ onto $S$. Then $\bar{\varphi}$ is an endomorphism of $S$. Since $S$ is a (finite dimensional) vector space over $Z / p Z$, we can write $\bar{\varphi}$ as the sum of two units. Let $\bar{\varphi}=\rho+\sigma$ where $\rho$ and $\sigma$ are automorphisms of $S$. Let $(\pi, \rho)$ denote the endomorphism of $A+S$ defined by: $(\pi, \rho)(a+s)=\pi(a)+\rho(s)$ if $a \in A$ and $s \in S$. We define $(\chi, \sigma+\varphi-\bar{\varphi})$ in a similar manner. Clearly, $(\pi, \rho)$ and $(\chi, \sigma+\varphi-\bar{\varphi})$ are automorphisms of $A+S$ and their sum is $\varphi$. Moreover, $(\pi, \rho)$ and $(\chi, \sigma+\varphi-\bar{\varphi})$ preserve heights in $G$; we verify, for example, that $(\chi, \sigma+\varphi-\bar{\varphi})$ is height preserving. Consider $a+s$ where $a \in A$ and $s \in S$. If $s=0$, then $(\chi, \sigma+\varphi-\bar{\varphi})(a+s)=\chi(a)$ and the height of $a+s=a$ is not altered since $\chi$ preserves heights. If $h_{G}(a)<\alpha+n$, then $h_{G}(a+s)=h_{G}(a)$ since $s \in p^{\alpha+n} G$. Hence $(\chi, \sigma+\varphi-\bar{\varphi})(a+s)=\chi(a)+\sigma(s)+\varphi(s)-\bar{\varphi}(s)$ has the same height in $G$ as $a+s$ since $\chi(a), a$ and $a+s$ all have the same height and since $\sigma(s), \varphi(s)$ and $\bar{\varphi}(s)$ all have greater height. We have shown that in order for $(\chi, \sigma+\varphi-\bar{\varphi})$ to alter the height of $a+s$ it must be the case that $s \neq 0$ and $h_{G}(a) \geqq \alpha+n$, so assume that $s \neq 0$ and that $h_{G}(a) \geqq \alpha+n$. Then the height of $a+s$ is exactly $\alpha+n$ and it is easy to verify that the height of $(\chi, \sigma+\varphi-\bar{\varphi})(a+s)$ is also exactly $\alpha+n$. Thus $(\chi, \sigma+\varphi-\bar{\varphi})$ preserves heights in $G$.

At this point, we have extended $\pi$ and $\chi$ to height-preserving automorphisms of $A+S$ such that the sum of the extensions is still $\varphi$. For simplicity of notation, we now consider the domain of $\pi$ and $\chi$ to be $A+S$ and we let $B_{0}=A+S$. Set $B$ $=\left\{p^{\alpha+n} G, A\right\}$. We wish to extend $\pi$ and $\chi$ to height-preserving automorphisms of $B$ in such a way that their sum is $\varphi$. Suppose that $B_{0} \subseteq B_{1} \subseteq B$ and that $\pi$ and $\chi$ have been extended to mappings of $B_{1}$ into $G$ in such a way that their sum is $\varphi$ on $B_{1}$ and the extensions continue to preserve heights in $G$. Observe that $\pi$ and $\chi$ must map $B_{1}$ into $B$ (but not necessarily $B_{1}$ ). Suppose that $B_{1} \neq B$. Choose $x$ in $B$ not in $B_{1}$. 
Since $x+B_{1}=x^{\prime}+B_{1}$ where $x^{\prime} \in p^{\alpha+n} G$, we assume that $x$ is in $p^{\alpha+n} G$. Note that $x+b \in p^{\alpha+n+1} G$ is impossible where $b \in B_{1}$ since $p^{\alpha+n+1} G \subseteq A \subseteq B_{1}$ and $x \notin B_{1}$. Also observe that $p x \in p^{\alpha+n+2} G$ implies that $x-a \in p^{\alpha+n} G[p] \subseteq B_{0} \subseteq B_{1}$ and that $x \in B_{1}$. Hence $h_{G}(p x)=\alpha+n+1$. Since $p x \in A$ and since $\pi$ is height preserving, $h_{G}(\pi(p x))=\alpha+n+1$. Thus there exists $y \in p^{\alpha+n} G$ such that $p y=\pi(p x)$.

We can extend $\pi$ to a height-preserving isomorphism of $\left\{B_{1}, x\right\}$ into $B$ by mapping $x$ onto $y$. Extend $\chi$ to $\left\{B_{1}, x\right\}$ by setting $\chi=\varphi-\pi$. Then $\chi$ maps $\left\{B_{1}, x\right\}$ into $B$ and is height preserving, by hypothesis, on $B_{1}$. In order to show that $\chi$ is height preserving on $\left\{B_{1}, x\right\}$, let $z=x+b$ where $b \in B_{1}$. If $h_{G}(b) \geqq \alpha+n$, then $h_{G}(z)=\alpha+n$ and $h_{G}(\chi(z))$ $=h_{G}(\varphi(x)-y+\chi(b)) \geqq \alpha+n$. Assume $h_{G}(\chi(z))>\alpha+n$. Then $p z \in p^{\alpha+n+2} G$ which as we have shown is impossible. Now consider the case where $h_{G}(b)<\alpha+n$. We have

$$
h_{G}(z)=h_{G}(b)=h_{G}(\chi(b))=h_{G}(\varphi(x)-y+\chi(b))=h_{G}(\chi(z)) \text {, }
$$

and conclude that $\chi$ is height preserving on $\left\{B_{1}, x\right\}$. It follows that $\pi$ and $\chi$ can be extended to height-preserving isomorphisms of $B$ into $B$ in such a way that their sum is $\varphi$. Since $B / A$ is finite, $\pi$ and $\chi$ are necessarily automorphisms of $B$. Since $p^{\alpha+n} G \subseteq B$, the theorem now follows by induction on $n$, the smallest nonnegative integer such that $p^{\alpha+n+1} G \subseteq A$.

3. In this section, Theorem 2.1 is generalized in several directions. In order to make these generalizations, we shall frequently need to consider subgroups with the following property.

Definition 3.1. A subgroup $A$ of the primary group $G$ is called a nice subgroup of $G$ if $p^{\alpha}(G \mid A)=\left\{p^{\alpha} G, A\right\} \mid A$ for each ordinal $\alpha$.

It was shown in [3] that if $G$ is totally projective, then there exists a collection $\mathscr{C}$ of nice subgroups of $G$ such that:

(0) $0 \in \mathscr{C}$.

(1) $\mathscr{C}$ is closed with respect to group-theoretic union.

(2) If $A \in \mathscr{C}$ and if $H$ is a subgroup of $G$ such that $\{A, H\} / A$ is countable, there exists $B \in \mathscr{C}$ such that $B \supseteq\{A, H\}$ and $B / A$ is countable.

For details concerning totally projective groups see [5] or [3].

The next theorem is of interest even when $\varphi=0$ in which case $p$ can be an arbitrary prime.

THEOREM 3.2. Let $G$ be a p-primary abelian group where $p \neq 2$ and let $A$ be a nice subgroup of $G$. Suppose that $\varphi$ is an endomorphism of $G$ that maps $A$ into $A$ and suppose that $\pi$ and $\chi$ are height-preserving automorphisms of $A$ such that $\pi+\chi=\varphi$ on $A$. Let $B$ be a finite extension of $A$ and suppose that $\pi$ and $\chi$ have been extended to height-preserving isomorphisms from $B$ into $G, B>B^{\prime}$ and $B \gg B^{\prime \prime}$, in such a way that $\pi+\chi=\varphi$ on $B$. If $F$ is any finite extension of $A$, then there exist finite extensions $C, C^{\prime}$, and $C^{\prime \prime}$ of $B, B^{\prime}$, and $B^{\prime \prime}$, respectively, all containing $F$ such that $\pi$ and $\chi$ can be extended to height-preserving isomorphisms $C \nrightarrow C^{\prime}$ and $C \nrightarrow C^{\prime \prime}$ in such a way that $\pi+\chi=\varphi$ on $C$. 
Proof. Since $B$ is a finite extension of a nice subgroup of $G, B$ is a nice subgroup of $G$. The same holds for $B^{\prime}$ and $B^{\prime \prime}$. Thus each coset $x+B$ contains an element that has maximal height. Such a distinguished element of a coset is called a proper element.

Suppose there exists $x$ in $F$ not in $B$. We shall choose such an $x$ with $p x \in B$. Moreover, since $B$ is a nice subgroup of $G$, we can choose $x$ proper with respect to $B$. Let $h_{G}(x)=\alpha$. Two cases are distinguished.

Case 1. $h_{G}(p(x+b))=\alpha+1$ for each $b \in B$ such that $x+b$ is a proper element of the coset $x+B$.

Since $\pi$ is height preserving, there exists $y$ in $G$ such that $h_{G}(y)=\alpha$ and $\pi(p x)=p y$. Extend $\pi$ and $\chi$ to $\{B, x\}$ by letting $\pi(x)=y$ and $\chi(x)=\varphi(x)-y$. Then $\pi$ and $\chi$ are height preserving and $\varphi=\pi+\chi$ on $\{B, x\}$.

Case 2. $h_{G}(p(x+b)) \geqq \alpha+2$ for some $b \in B$ such that $x+b$ is a proper element of the coset $x+B$.

In Case 2, we can choose $x$ such that $h_{G}(p x) \geqq \alpha+2$. Then $p x=p g$ where $g \in p^{\alpha+1} G$. Note that $x-g$ is in $G[p]$ and is proper with respect to $B$. Since $\pi: B \succ B^{\prime}$ is height preserving and since $B$ and $B^{\prime}$ are finite extensions of $A$, there exists $z_{1}$ in $G[p]$ having height $\alpha$ and proper with respect to $B^{\prime}$. Similarly, there exists $z_{2}$ in $G[p]$ having height $\alpha$ and proper with respect to $B^{\prime \prime}$. Observe that one of $z_{1}, z_{2}$ and $z_{1}+z_{2}$ has height $\alpha$ and is proper with respect to both $B^{\prime}$ and $B^{\prime \prime}$, so choose $z$ in $G[p]$ that has height $\alpha$ and is proper with respect to both $B^{\prime}$ and $B^{\prime \prime}$. Let $w \in p^{\alpha+1} G$ be such that $\pi(p x)=p w$. Set $y=w+z$. We can extend $\pi$ to a height-preserving isomorphism from $\{B, x\}$ into $G$ by mapping $x$ onto $y$. Furthermore, if $\varphi-\pi$ does not preserve heights on $\{B, x\}$, we can make $\varphi-\pi$ preserve heights by changing the definition of $y$ to $y=w+2 z$-this change is perfectly acceptable since $p \neq 2$.

We have shown that we can extend $\pi$ and $\chi$ in the desired way to a common domain containing $x$, but we need to show now that we can extend so that $x$ is also contained in the images of $\pi$ and $\chi$. First, we concentrate on $\pi$. As usual, there is no loss of generality in assuming that $p x \in B^{\prime}, x \notin B^{\prime}$, and that $x$ is proper with respect to $B^{\prime}$. As before, there are two cases.

Case 1 . $h_{G}\left(p\left(x+b^{\prime}\right)\right)=\alpha+1$ for each $b^{\prime} \in B^{\prime}$ such that $x+b^{\prime}$ is a proper element of the coset $x+B^{\prime}$.

There exists $y \in G$ such that $h_{G}(y)=\alpha$ and $\pi(p y)=p x$. We can extend $\pi$ to $\{B, y\}$ by mapping $y$ onto $x$. This extension is height preserving and it is easy to verify that $\varphi-\pi$ is also height preserving.

The next case is the most difficult part of the theorem.

Case 2. $h_{G}\left(p\left(x+b^{\prime}\right)\right) \geqq \alpha+2$ for some $b^{\prime} \in B^{\prime}$ such that $x+b^{\prime}$ is a proper element of the coset $x+B^{\prime}$.

In this case we can choose $x$ such that $h_{G}(p x) \geqq \alpha+2$. Since $p x=p g$ where $g \in p^{\alpha+1} G$, it follows that $x-g$ has order $p$, height $\alpha$, and is proper with respect to $B^{\prime}$. Thus there exists $z$ in $G[p]$ having height $\alpha$ which is proper with respect to $B$. Choose $w \in p^{\alpha+1} G$ such that $\pi(p w)=p x$ and let $y=w+z$. We can extend $\pi$ to a 
height-preserving isomorphism, that contains $x$ in its image, by mapping $y$ onto $x$. However, $\varphi-\pi$ may not preserve heights; $\varphi-\pi$ fails to preserve heights if and only if

$$
\varphi(z)-x+\chi\left(b_{0}\right) \in p^{\alpha+1} G \text { for some } b_{0} \in B .
$$

Before, the situation was remedied by changing the definition of $y$ to $w+2 z$. Unfortunately, the same change now does not necessarily remove the defect; we can have both (1) and

$$
2 \varphi(z)-x+\chi\left(b_{1}\right) \in p^{\alpha+1} G \text { for some } b_{1} \in B .
$$

However, (1) and (2) immediately yield

$$
\varphi(z)+\chi\left(b_{1}-b_{0}\right) \in p^{\alpha+1} G
$$

and

$$
x+\chi\left(b_{1}-2 b_{0}\right) \in p^{\alpha+1} G .
$$

Setting $b_{2}=2 b_{0}-b_{1}$, we have from (4) and the fact that $\chi=\varphi-\pi$ the relation

$$
x+\pi\left(b_{2}\right)-\varphi\left(b_{2}\right) \in p^{\alpha+1} G .
$$

Observe that $h_{G}\left(b_{2}\right) \geqq \alpha$ since $h_{G}\left(b_{i}\right) \geqq \alpha$ for $i=1,2$. Furthermore, $h_{G}\left(p b_{2}\right) \geqq \alpha+2$ since $p x \in p^{\alpha+2} G$ and $p z=0$. Let

$$
p^{\alpha} G[p]=S_{\alpha}+p^{\alpha+1} G[p] .
$$

All the time we have known that there exists an element in $S_{\alpha}$ that is proper with respect to $B^{\prime}$, but (5) tells us more. Let $E_{\alpha}=\rho \varphi\left(S_{\alpha}\right)$, where $\rho$ is the natural projection of $p^{\alpha} G[p]$ onto $S_{\alpha}$, associated with the decomposition (6). For any subgroup $H$ of $G$, define

$$
E_{\alpha}(H)=\left[e \in E_{\alpha}: e+h \in p^{\alpha+1} G \text { for some } h \in H\right] .
$$

From (5) and the fact that $x$ (and therefore $x+\pi\left(b_{2}\right)$ ) is proper with respect to $B^{\prime}$, we conclude that $E_{\alpha} / E_{\alpha}\left(B^{\prime}\right) \neq 0$. We shall borrow from Lemma 3.3 the result that $E_{\alpha} / E_{\alpha}\left(B^{\prime}\right) \cong E_{\alpha} / E_{\alpha}\left(B^{\prime \prime}\right)$. Hence there exists $e$ in $E_{\alpha}$ not in $E_{\alpha}\left(B^{\prime \prime}\right)$. However, by the definition of $E_{\alpha}$, we have $e=\rho \varphi\left(z^{\prime}\right)$ for some $z^{\prime} \in S_{\alpha}$. Since $e \notin E_{\alpha}\left(B^{\prime \prime}\right), \varphi\left(z^{\prime}\right)+b^{\prime \prime}$ $\notin p^{\alpha+1} G$ if $b^{\prime \prime} \in B^{\prime \prime}$. Recall that $z \in p^{\alpha+1} G[p]$ was chosen proper with respect to $B$ and has height $\alpha$. We may of course assume that $z \in S_{\alpha}$. Observe that at least one of $z, z^{\prime}$ and $z+z^{\prime}$ is proper with respect to $B$ and has the property that its image under $\varphi$ is proper with respect to $B^{\prime \prime}$. Hence we may assume that $z \in S_{\alpha}$ is proper with respect to $B$ and that $\varphi(z)$ is proper with respect to $B^{\prime \prime}$. However, with this choice of $z$, we find that (3) is impossible, so we can extend $\pi$ by mapping $w+z$ onto $x$ or we can extend by mapping $w+2 z$ onto $x$. One or the other makes $\varphi-\pi$ height preserving and $\pi$ is height preserving in either case. Since $\pi$ and $\chi$ are symmetric with respect to the hypotheses, the proof of the theorem is finished.

LEMMA 3.3. $E_{\alpha} / E_{\alpha}\left(B^{\prime}\right) \cong E_{\alpha} / E_{\alpha}\left(B^{\prime \prime}\right)$, the notation and hypotheses being the same as in the proof of Theorem 3.2.

Proof. For each $x \in E_{\alpha}\left(B^{\prime}\right)$, there exists $b \in B$ such that $x+\pi(b) \in p^{\alpha+1} G$. Since $\pi=\varphi-\chi$, we have $x+\varphi(b)-\chi(b) \in p^{\alpha+1} G$. Since $p x=0$ and since $\pi$ preserves 
heights, $p b \in p^{\alpha+2} G$. Let $p b=p w$ where $w \in p^{\alpha+1} G$. Observe that $b-w \in p^{\alpha} G[p]$ $=S_{\alpha}+p^{\alpha+1} G[p]$. Let $\varphi(b-w)=s+t$ where $s \in S_{\alpha}$ and $t \in p^{\alpha+1} G[p]$. We note that $s$ is uniquely determined by $x$ and that $\varphi(b)-s \in p^{\alpha+1} G$. Denoting $s$ by $\psi(x)$, we have that $x+\psi(x)-\chi(b) \in p^{\alpha+1} G$. Clearly, $\psi(x) \in \rho \varphi\left(S_{\alpha}\right)=E_{\alpha}$, so $x+\psi(x) \in E_{\alpha}\left(B^{\prime \prime}\right)$. The mapping $x \rightarrow x+\psi(x)$ is an isomorphism of the vector space $E_{\alpha}\left(B^{\prime}\right)$ onto $E_{\alpha}\left(B^{\prime \prime}\right)$ that takes the subspace $E_{\alpha}(A)$ onto itself. Since $B^{\prime}$ and $B^{\prime \prime}$ are finite over $A$, it quickly follows that $E_{\alpha}\left(B^{\prime}\right)$ and $E_{\alpha}\left(B^{\prime \prime}\right)$ are finite over $E_{\alpha}(A)$. Thus $E_{\alpha}\left(B^{\prime}\right) / E_{\alpha}(A)$ and $E_{\alpha}\left(B^{\prime \prime}\right) / E_{\alpha}(A)$ have the same finite dimension, and we conclude that $E_{\alpha} / E_{\alpha}\left(B^{\prime}\right)$ $\cong E_{\alpha} / E_{\alpha}\left(B^{\prime \prime}\right)$.

Our main result is the following

THEOREM 3.4. Suppose that $G$ is a p-primary abelian group with $p \neq 2$. Let $H$ be a nice subgroup of $G$ and let $\varphi$ be an endomorphism of $G$ that maps $H$ into itself. If the restriction of $\varphi$ to $H$ is the sum of two automorphisms of $H$ that preserve heights in $G$, then $\varphi$ is the sum of two automorphisms of $G$ provided that $G / H$ is totally projective.

Proof. Let $\mathscr{C}$ be a collection of nice subgroups of $G / H$ that satisfy conditions (0)-(2); recall the introduction to this section. Consider the family $\mathscr{F}$ of subgroups $A$ of $G$ such that $H \subseteq A \subseteq G$ and $A / H \in \mathscr{C}$. Let $\mathscr{E}$ denote the set of extensions $\pi^{+}$ of $\pi$ with the property that $\pi^{+}$and $\varphi-\pi^{+}$both are automorphisms of some $A$ in $\mathscr{F}$ that preserve heights in $G$. The set $\mathscr{E}$ can be ordered in the natural way; one element of $\mathscr{E}$ is greater than another if the first is an extension of the second. Since Zorn's lemma is applicable there is a maximal element $\pi^{+}$of $\mathscr{E}$, which we shall continue to denote by $\pi$. Let $\pi: A \gg A$ and set $\chi=\varphi-\pi: A \gg A$. Since $A / H$ is a nice subgroup of $G / H$, it follows that $A$ is a nice subgroup of $G$.

Suppose that $A \neq G$ and let $x$ in $G$ be outside of $A$. By Theorem 3.2, there exist finite extensions $B_{1} \gg B_{1}^{\prime}$ and $B_{1} \gg B_{1}^{\prime \prime}$ of $\pi$ and $\chi$, respectively, that preserve heights and such that the sum is $\varphi$ on $B_{1}$ where $x \in B_{1}$. Repeated applications of Theorem 3.2 yield ascending sequences of such finite extensions $B_{n} \gg B_{n}^{\prime}$ and $B_{n} \gg B_{n}^{\prime \prime}$ with the property that

$$
B_{n+1} \cap B_{n+1}^{\prime} \cap B_{n+1}^{\prime \prime} \supseteq B_{n} \cup B_{n}^{\prime} \cup B_{n}^{\prime \prime} .
$$

In particular, $\bigcup B_{n}=\bigcup B_{n}^{\prime}=\bigcup B_{n}^{\prime \prime}, n<\omega$. Moreover, using condition (2) on the collection $\mathscr{C}$, we can force $\left(\bigcup_{n<\omega} B_{n}\right) / H$ to be a member of $\mathscr{C}$. This gives us an element of $\mathscr{E}$ still larger than our maximal element, so we conclude that $A=G$. This concludes the proof of the theorem.

4. In this section we give a few applications of Theorem 3.4. Taking $H=0$ in Theorem 3.4, we obtain

THEOREM 4.1. A totally projective $p$-group, $p \neq 2$, has the property that any endomorphism is the sum of two automorphisms.

COROLlARY 4.2 (CASTAGNA). If the primary group $G$ is a direct sum of countable groups and has an odd prime associated with it, then any endomorphism of $G$ is the sum of two automorphisms. 
A trivial corollary of Theorem 4.1 is the following

COROLLARY 4.3. $E(G)=\{A(G)\}$ if $G$ is a totally projective $p$-group with $p \neq 2$.

More along the line of [4], we have

COROllary 4.4. If $G$ is p-primary and if $G / p^{\alpha} G$ is totally projective for some ordinal $\alpha$, then any automorphism of $p^{\alpha} G$ can be extended to an automorphism of $G$.

The reason that no restriction on $p$ is necessary in Corollary 4.4 is that no restriction is necessary for Theorem 3.2 in case $\varphi=0$.

COROLlaRy 4.5. Let $G$ be a p-primary group with $p \neq 2$ and let $\alpha$ be an ordinal number such that $G / p^{\alpha} G$ is totally projective. Then $E(G)=\{A(G)\}$ if and only if $E\left(p^{\alpha} G\right)=\left\{A\left(p^{\alpha} G\right)\right\}$.

Proof. The proof is trivial in one direction (without Theorem 3.4) since $p^{\alpha} G$ is fully invariant. In order to show that $E\left(p^{\alpha} G\right)=\left\{A\left(p^{\alpha} G\right)\right\}$ implies $E(G)=\{A(G)\}$, let $\varphi \in E(G)$ and assume that $E\left(p^{\alpha} G\right)=\left\{A\left(p^{\alpha} G\right)\right\}$. Let $\varphi_{\alpha}$ be the restriction of $\varphi$ to $p^{\alpha} G$. Write $\varphi_{\alpha}=\sum \pi_{\alpha, i}$ where $\pi_{\alpha, i}$ is an automorphism of $p^{\alpha} G$. By Corollary 4.4, $\pi_{\alpha, i}$ can be extended to an automorphism $\pi_{i}$ of $G$ for each $i$. Now $\rho=\varphi-\sum \pi_{i}$ is an endomorphism of $G$ that is zero on $p^{\alpha} G$. Hence $\rho$ is the sum of two automorphisms on $p^{\alpha} G$ and therefore $\rho$ is the sum of two automorphisms on $G$. Write $\rho=\pi+\chi$ where $\pi$ and $\chi$ are automorphisms of $G$. Then $\varphi=\sum \pi_{i}+\pi+\chi$ is a sum of automorphisms.

Almost the same argument can be used to establish the following result; for definition of large subgroup, see [6].

COROLlaRY 4.6. Let $G$ be a p-primary group with $p \neq 2$ and let $L$ be a large subgroup of $G$. Then $E(G)=\{A(G)\}$ if and only if $E(L)=\{A(L)\}$.

\section{REFERENCES}

1. F. Castagna, Sums of automorphisms of a primary abelian group, (to appear).

2. H. Freeman, On endomorphisms of primary abelian groups, J. London Math. Soc. 43 (1968), 305-307.

3. P. Hill, On the classification of abelian groups, (to appear).

4. P. Hill and C. Megibben, Extending automorphisms and lifting decompositions in abelian groups, Math. Ann. 175 (1968), 159-168.

5. R. Nunke, Homology and direct sums of countable groups, Math. Z. 101 (1967), 182-212.

6. R. S. Pierce, "Homomorphisms of primary groups," in Topics in Abelian groups, Scott Foresman and Co., Glenville, Illinois, 1963.

7. - Endomorphism rings of primary abelian groups, Proc. Colloq. Abelian Groups, Budapest, 1964, 125-137.

8. R. W. Stringall, Endomorphism rings of abelian groups generated by automorphism groups, Acta. Math. 18 (1967), 401-404.

UNIVERSITY OF HOUSTON, Houston, Texas

The Florida State University, Tallahassee, Florida 